\title{
Generating functions attached to some infinite matrices
}

\author{
Paul Monsky \\ Brandeis University \\ Waltham MA 02454-9110, USA \\ monsky@brandeis.edu
}

Submitted: Aug 9, 2010; Accepted: Dec 13, 2010; Published: Jan 5, 2011

Mathematics Subject Classification: 05E40 and 05A15

\begin{abstract}
Let $V$ be an infinite matrix with rows and columns indexed by the positive integers, and entries in a field $F$. Suppose that $v_{i, j}$ only depends on $i-j$ and is 0 for $|i-j|$ large. Then $V^{n}$ is defined for all $n$, and one has a "generating function" $G=\sum a_{1,1}\left(V^{n}\right) z^{n}$. Ira Gessel has shown that $G$ is algebraic over $F(z)$. We extend his result, allowing $v_{i, j}$ for fixed $i-j$ to be eventually periodic in $i$ rather than constant. This result and some variants of it that we prove will have applications to Hilbert-Kunz theory.
\end{abstract}

\section{Introduction}

Throughout, $\Lambda$ is a ring with identity element 1 . Suppose that $w_{i, j}, i$ and $j$ ranging over the positive integers, are in $\Lambda$ and that $w_{i, j}=0$ whenever $i-j$ lies outside a fixed finite set. Then if $W$ is the infinite matrix $\left|w_{i, j}\right|$, one may speak of $W^{n}$ for all $n \geq 0$, and one gets a generating function $G(W)=\sum_{0}^{\infty} a_{n} z^{n}$ in $\Lambda[[z]]$, where $a_{n}$ is the $(1,1)$ entry in the matrix $W^{n}$. We shall prove:

Theorem I. Suppose that $w_{i, j}=0$ if $i-j \notin\{-1,0,1\}$, and that $w_{i+1, j+1}=w_{i, j}$ unless $i=j=1$. Suppose further that $\Lambda=M_{s}(F), F$ a field, so that $G(W)$ may be viewed as an $s$ by $s$ matrix with entries in $F[[z]]$. Then these matrix entries are algebraic over $F(z)$.

Corollary. Let $F$ be a field and $v_{i, j}, i$ and $j$ ranging over the positive integers, be in $F$. Suppose:

(a) $v_{i, j}=0$ whenever $i-j$ lies outside a fixed finite set.

(b) For fixed $r$ in $Z, v_{i, i+r}$ is an eventually periodic function of $i$.

Then if $V$ is the matrix $\left|v_{i, j}\right|$, the generating function $G(V)$ is algebraic over $F(z)$. 
Proof. To derive the corollary we choose $s$ so that:

(1) $v_{i, j}=0$ whenever $i \leq s$ and $j>2 s$ or $j \leq s$ and $i>2 s$.

(2) $v_{i+s, j+s}=v_{i, j}$ whenever $i+j \geq s+2$.

We then write the initial $2 s$ by $2 s$ block in $V$ as $|\underset{A}{D} \underset{B}{C}|$ with $A, B, C, D$ in $M_{s}(F)$. Our choice of $s$ tells us that $V$ is built out of $s$ by $s$ blocks, where the blocks along the diagonal are a single $D$, followed by $B$ 's, those just below a diagonal block are $A$ 's, those just above a diagonal block are $C$ 's, and all other entries are 0 . Now let $\Lambda=M_{s}(F)$ and $W=\left|w_{i, j}\right|$ where $w_{i+1, i}=A, w_{i, i+1}=C, w_{1,1}=D, w_{i, i}=B$ for $i>1$, and all other $w_{i, j}$ are 0 . View $G(W)$ as an $s$ by $s$ matrix with entries in $F[[z]]$. One sees easily that $G(V)$ is the $(1,1)$ entry in this matrix, and Theorem I applied to $W$ gives the corollary.

Remark. When $v_{i, j}$ only depends on $i-j$, the above corollary is due to Gessel. (When the matrix entries of $V$ are all 0 's and 1 's the result is contained in Corollary 5.4 of [1]. The restriction on the matrix entries isn't essential in Gessel's proof, as one can use a generating function for walks with weights.)

Our proof of Theorem I is easier than Gessel's proof of his special case of the corollary. The reason for this is that by working over $\Lambda$ rather than over $F$ we are able to restrict our study to walks with step-sizes in $\{-1,0,1\}$. (A complication, fortunately minor, is that the weights must be taken in the non-commutative ring $\Lambda$.) Our proof is well-adapted to finding an explicit polynomial relation between $G(V)$ and $z$; we'll work out a few examples. This paper would not have been possible without Ira Gessel's input. I thank him for showing me tools of the combinatorial trade.

\section{Walks and generating functions}

Definition 2.1. If $l \geq 0$, an ordered $l+1$-tuple $\alpha=\left(\alpha_{0}, \ldots, \alpha_{l}\right)$ of integers is a (Motzkin) walk of length $l=l(\alpha)$ if each of $\alpha_{1}-\alpha_{0}, \ldots, \alpha_{l}-\alpha_{l-1}$ is in $\{-1,0,1\}$.

We say that the start of the walk is $\alpha_{0}$, the finish is $\alpha_{l}$, and that $\alpha$ is a walk from $\alpha_{0}$ to $\alpha_{l}$.

Definition 2.2. If $\alpha$ and $\beta$ are walks of lengths $l$ and $m$, the concatenation $\alpha \beta$ of $\alpha$ and $\beta$ is the walk $\left(\alpha_{0}, \ldots, \alpha_{l}, \alpha_{l}+\left(\beta_{1}-\beta_{0}\right), \ldots, \alpha_{l}+\left(\beta_{m}-\beta_{0}\right)\right)$ of length $l+m$.

Now let $\Lambda$ be a ring with identity element 1 , and $A, B, C, D$ lie in $\Lambda$. To each walk $\alpha$ we attach weights $w(\alpha)$ and $w^{*}(\alpha)$ in $\Lambda$ :

Definition 2.3. If $l(\alpha)=0, w(\alpha)=w^{*}(\alpha)=1$. If $l(\alpha)>0, w(\alpha)=U_{1} \cdot \ldots \cdot U_{l}$ where $U_{i}=A, B$ or $C$ according as $\alpha_{i}-\alpha_{i-1}$ is -1 , 0 , or 1 . The definition of $w^{*}(\alpha)$ is the same with one change: if $\alpha_{i}=\alpha_{i-1}=0$ then $U_{i}=D$ rather than $B$.

Evidently $w(\alpha \beta)=w(\alpha) w(\beta)$. Furthermore $w^{*}(\alpha \beta)=w^{*}(\alpha) w^{*}(\beta)$ whenever $\alpha$ and $\beta$ are walks from 0 to 0 . 
Definition 2.4. $\alpha$ is "standard" if each $\alpha_{i} \geq \alpha_{l}$. Note that a walk from 0 to 0 is standard if and only if each $\alpha_{i} \geq 0$.

Definition 2.5. $\alpha$ is "primitive" if $l(\alpha)>0, \alpha_{0}=\alpha_{l}$ and no $\alpha_{i}$ with $0<i<l$ is $\alpha_{0}$. Note that a standard walk from 0 to 0 is primitive if and only if $l(\alpha)>0$ and each $\alpha_{i}$, $0<i<l$, is $>0$.

\section{Definition 2.6.}

(1) $G(w)=\sum w(\alpha) z^{l(\alpha)}$, the sum extending over all standard walks from 0 to $0 . H(w)$ is the sum extending over all primitive standard walks from 0 to 0.

(2) $G\left(w^{*}\right)$ and $H\left(w^{*}\right)$ are defined similarly, using $w^{*}(\alpha)$ in place of $w(\alpha)$.

Lemma 2.7. Let $G=G(w), H=H(w)$. Then, in $\Lambda[[z]]$ :

(1) $G=1+H+H^{2}+\cdots$

(2) $H=B z+C G A z^{2}$

Proof. Every standard walk from 0 to 0 of length $>0$ is either primitive or uniquely a concatenation of two or more primitive standard walks from 0 to 0 . The multiplicative property of $w$ now gives (1). To prove (2) note that the primitive standard walk $(0,0)$ has $w=B$. And a primitive standard walk from 0 to 0 of length $l>1$ is a concatenation of $(0,1)$, a standard walk, $\beta$, from 0 to 0 of length $l-2$ and $(0,-1)$. Then $w(\alpha)=C w(\beta) A$. Since $\alpha \rightarrow \beta$ gives a 1-1 correspondence between primitive standard walks of length $l$ from 0 to 0 and standard walks of length $l-2$ from 0 to 0 , we get the result.

Corollary 2.8. If $G=G(w)$, then $G-1-(B G) z-(C G A G) z^{2}=0$ in $\Lambda[[z]]$.

Proof. By (1) of Lemma 2.7, $(1-H) \cdot G=1$. Substituting $H=B z+C G A z^{2}$ gives the result.

Theorem 2.9. Suppose that $\Lambda=M_{s}(F), F$ a field, so that $G(w)$ may be viewed as an $s$ by $s$ matrix with entries in $F[[z]]$. Then these matrix entries, $u_{i, j}$, are algebraic over $F(z)$.

Proof. Let $U=\left|U_{i, j}\right|$ be an $s$ by $s$ matrix of indeterminates over $F$, and $p_{i, j}$ be the $(i, j)$ entry in $U-I_{s}-(B U) z-(C U A U) z^{2}$. The $p_{i, j}$ are degree 2 polynomials in $U_{1,1}, \ldots, U_{s, s}$ with coefficients in $F[z]$. By Corollary $2.8, p_{i, j}\left(u_{1,1}, \ldots, u_{s, s}\right)=0$. Now $p_{i, j}=U_{i, j}-$ $\delta_{i, j}-z f_{i, j}\left(U_{1,1}, \ldots, U_{s, s}, z\right)$ where the $f_{i, j}$ are polynomials with coefficients in $F$. It follows that the Jacobian matrix of the $p_{i, j}$ with respect to the $U_{i, j}$, evaluated at $\left(u_{1,1}, \ldots, u_{s, s}\right)$, is congruent to $I_{s^{2}} \bmod z$ in the $s^{2}$ by $s^{2}$ matrix ring over $F[[z]]$, and so is invertible. Thus $\left(u_{1,1}, \ldots, u_{s, s}\right)$ is an isolated component of the intersection of the hypersurfaces $p_{i, j}\left(U_{1,1}, \ldots, U_{s, s}\right)=0$, and so its co-ordinates, $u_{1,1}, \ldots, u_{s, s}$, are algebraic over $F(z)$. 
Remark. We sketch a proof, based on the Nullstellensatz and Nakayama's Lemma, of the result from algebraic geometry used in the last sentence above. Suppose then that $K \subset L$ are fields, that $f_{1}, \ldots, f_{n}$ are in $K\left[x_{1}, \ldots, x_{n}\right]$, and that $a_{1}, \ldots, a_{n}$ are in $L$. Suppose further that each $f_{i}\left(a_{1}, \ldots, a_{n}\right)=0$, and that $J\left(a_{1}, \ldots, a_{n}\right) \neq 0$, where $J$ is the Jacobian determinant of the $f_{i}$ with respect to the $x_{j}$. We shall show that each $a_{i}$ is algebraic over $K$. We may assume that $K$ is algebraically closed. The kernel of evaluation at $\left(a_{1}, \ldots, a_{n}\right)$ is a prime ideal, $P$, of $K\left[x_{1}, \ldots, x_{n}\right]$. Each $f_{i}$ is in $P$ and $J$ is not in $P$. By the Nullstellensatz, $P \subset$ some $m=\left(x_{1}-b_{1}, \ldots, x_{n}-b_{n}\right)$ with $J\left(b_{1}, \ldots, b_{n}\right) \neq 0$. Each $f_{i}$ is in $m$. Writing $f_{i}$ as a polynomial in $x_{1}-b_{1}, \ldots, x_{n}-b_{n}$, and using the fact that $J\left(b_{1}, \ldots, b_{n}\right) \neq 0$, we find that $\left(P, m^{2}\right)=m$. Now $P$ is prime, and it follows from Nakayama's Lemma that $P=m$. So $a_{i}=b_{i}$, and is in $K$.

Lemma 2.10. $G\left(w^{*}\right)^{-1}-G(w)^{-1}=(B-D) z$.

Proof. The proof of Lemma 2.7 (1) shows that $G\left(w^{*}\right)^{-1}=1-H\left(w^{*}\right)$ with $H\left(w^{*}\right)$ as in Definition 2.6. So it suffices to show that $H(w)-H\left(w^{*}\right)=(B-D) z$. Now for a primitive walk $\alpha$ of length $>1$ from 0 to 0 one cannot have $\alpha_{i-1}=\alpha_{i}=0$, and so $w(\alpha)=w^{*}(\alpha)$. On the other hand, for the primitive walk $(0,0), w=B$ and $w^{*}=D$. This gives the lemma.

Combining Lemma 2.10 with Theorem 2.9 we get:

Theorem 2.11. If $\Lambda=M_{s}(F)$ the matrix entries of the s by s matrix $G\left(w^{*}\right)$ are algebraic over $F(z)$.

Now let $W=\left|w_{i, j}\right|$ where $w_{i+1, i}=A, w_{i, i+1}=C, w_{1,1}=D, w_{i, i}=B$ for $i>1$, and all the other $w_{i, j}=0$. In view of Theorem 2.11 the proof of Theorem I will be complete once we show that $G(W)=G\left(w^{*}\right)$ where $w^{*}$ is the weight function of Definition 2.3. The key to this is:

Lemma 2.12. For $k \geq 1$ let $u_{k}^{(n)}$ be $\sum w^{*}(\alpha)$, the sum extending over all standard walks of length $n$ from $k-1$ to 0 . Then:

(1) $u_{k}^{(0)}=1$ or 0 according as $k=1$ or $k>1$.

(2) $u_{1}^{(n+1)}=D u_{1}^{(n)}+C u_{2}^{(n)}$.

(3) If $k>1, u_{k}^{(n+1)}=A u_{k-1}^{(n)}+B u_{k}^{(n)}+C u_{k+1}^{(n)}$.

Lemma 2.12 has the following immediate corollaries, with the first proved by induction on $n$.

Corollary 2.13. The first column vector in $W^{n}$ is $\left(u_{1}^{(n)}, u_{2}^{(n)}, \ldots\right.$

Corollary 2.14. The $(1,1)$ coefficient of $W^{n}$ is $\sum w^{*}(\alpha)$, the sum extending over all standard walks of length $n$ from 0 to 0 . So $G(W)=G\left(w^{*}\right)$. 
It remains to prove Lemma 2.12. (1) is evident. Let $\alpha$ be a standard walk of length $n$ from 0 or 1 to 0 . Then $\beta=\left(0, \alpha_{0}, \ldots, \alpha_{n}\right)$ is a standard walk of length $n+1$ from 0 to 0 , and $w^{*}(\beta)$ is $D w^{*}(\alpha)$ in the first case and $C w^{*}(\alpha)$ in the second. Also each standard walk $\beta$ of length $n+1$ from 0 to 0 arises in this way from some $\alpha$; explicitly $\alpha=\left(\beta_{1}, \ldots, \beta_{n}\right)$. Summing over $\beta$ we get (2). Similarly, suppose that $k>1$ and that $\alpha$ is a standard walk of length $n$ from $k-2, k-1$ or $k$ to 0 . Then $\beta=\left(k-1, \alpha_{0}, \ldots, \alpha_{n}\right)$ is a standard walk of length $n+1$ from $k-1$ to 0 and $w^{*}(\beta)=A w^{*}(\alpha)$ in the first case, $B w^{*}(\alpha)$ in the second, and $C w^{*}(\alpha)$ in the third. Also, each standard walk $\beta$ of length $n+1$ arises from such an $\alpha$; explicitly $\alpha=\left(\beta_{1}, \ldots, \beta_{n}\right)$. Summing over $\beta$ we get $(3)$, completing the proof.

Remark 2.15. To calculate the matrix entries of $G(W)$ explicitly as algebraic functions of $z$ by the method of Theorem 2.9 involves solving a system of $s^{2}$ quadratic equations in $s^{2}$ variables. This isn't practical when $s>2$; in the next section we give a different proof of Theorem 2.9 that is often better adapted to explicit calculations.

\section{A partial fraction proof of Theorem 2.9}

Theorem 3.1. $\sum w(\alpha) x^{\alpha_{0}}$, the sum extending over all length $n$ walks (not necessarily standard) with finish 0 , is the element $\left(A x+B+C x^{-1}\right)^{n}$ of $\Lambda\left[x, x^{-1}\right]$.

Proof. Denote the sum by $f_{n}$. Since $f_{0}=1$ it's enough to show that $f_{n+1}=(A x+B+$ $\left.C x^{-1}\right) f_{n}$. Let $v_{k}^{(n)}$ be the coefficient of $x^{k}$ in $f_{n}$. Then $v_{k}^{(n)}=\sum w(\alpha)$, the sum extending over all length $n$ walks from $k$ to 0 . The proof of (3) of Lemma 2.12, using all walks rather

than all standard walks, shows that $v_{k}^{(n+1)}=A v_{k-1}^{(n)}+B v_{k}^{(n)}+C v_{k+1}^{(n)}$ for all $k$ in $Z$, giving the result.

\section{Definition 3.2.}

$M_{0}(w)=\sum w(\alpha) z^{l(\alpha)}$, the sum extending over all 0 to 0 walks.

$M_{-1}(w)$ is the sum extending over all -1 to 0 (or 0 to 1 ) walks.

$M_{1}$ is the sum extending over all 1 to 0 (or 0 to -1) walks.

We'll generally omit the $w$ and just write $M_{0}, M_{-1}$ or $M_{1}$.

Corollary 3.3. Suppose that $i=0,-1$ or 1 . Then $M_{i}$ is the coefficient of $x^{i}$ in the element $\sum_{0}^{\infty}\left(A x+B+C x^{-1}\right)^{n} z^{n}$ of $\Lambda\left[x, x^{-1}\right][[z]]$.

Definition 3.4. $J_{0}=J_{0}(w)$ is $\sum w(\alpha) z^{l(\alpha)}$, the sum extending over all primitive 0 to 0 walks.

Theorem 3.5.

(1) $M_{0}=1+J_{0}+J_{0}^{2}+\cdots$.

(2) $G(w)=M_{0}-M_{1} M_{0}^{-1} M_{-1}$. 
Proof. (1) follows from the multiplicative property of $w$, as in the proof of Lemma 2.7. So $M_{0}^{-1}=1-J_{0}$, and (2) asserts that $G(w)=M_{0}+M_{1} J_{0} M_{-1}-M_{1} M_{-1}$. If $\alpha$ is a walk from 0 to 0 let $r(\alpha)$ be the number of ways of writing $\alpha$ as a concatenation of a walk from 0 to -1 and a walk from -1 to 0 . Also let $r_{1}(\alpha)$ be the number of ways of writing $\alpha$ as a concatenation of a walk from 0 to -1 , a primitive walk from -1 to -1 and a walk from -1 to 0 . The multiplicative property of $w$ shows that $M_{0}+M_{1} J_{0} M_{-1}-M_{1} M_{-1}=$ $\sum w(\alpha)\left(1+r_{1}(\alpha)-r(\alpha)\right) z^{l(\alpha)}$, the sum extending over all walks from 0 to 0 . If $\alpha$ is standard, $r_{1}(\alpha)=r(\alpha)=0$. If $\alpha$ is not standard there is an $i$ with $\alpha_{i}=-1$. Let $i_{1}<i_{2}<\cdots<i_{r}$ be those $i$ with $\alpha_{i}=-1$. One sees immediately that $r(\alpha)=r$ and that $r_{1}(\alpha)=r-1$. So $M_{0}+M_{1} J_{0} M_{-1}-M_{1} M_{-1}$ is the sum over the standard walks from 0 to 0 of $w(\alpha) z^{l(\alpha)}$, and this is precisely $G(w)$.

Suppose now that $\Lambda=M_{s}(F), F$ a field, so that $M_{0}, M_{1}$ and $M_{-1}$ may be viewed as $s$ by $s$ matrices with entries in $F[[z]]$. Theorem 3.5, (2), will give a new proof of Theorem 2.9 once we show that these matrix entries are algebraic over $F(z)$. The facts about the matrix entries of $M_{0}, M_{1}$ and $M_{-1}$ follow from a standard partial fraction decomposition argument - we'll give our own version.

The algebraic closure of the field of fractions of $F[[z]]$ is a valued field with value group $Q$. Let $\Omega$ be the completion of this field and ord : $\Omega \rightarrow Q \cup\{\infty\}$ be the ord function in $\Omega$. Let $\Omega^{\prime}$ consist of formal power series $\sum_{-\infty}^{\infty} a_{i} x^{i}$ with $a_{i} \in \Omega$ and ord $a_{i} \rightarrow \infty$ as $|i| \rightarrow \infty$. $\Omega^{\prime}$ has an obvious multiplication and is an overring of $F\left[x, x^{-1}\right][[z]] . l_{0}, l_{1}$ and $l_{-1}$ are the $\Omega$-linear maps $\Omega^{\prime} \rightarrow \Omega$ taking $\sum a_{i} x^{i}$ to $a_{0}, a_{1}$ and $a_{-1}$. Note that $\overline{F(z)}$, the algebraic closure of $F(z)$, imbeds in $\Omega$.

Lemma 3.6. Suppose $\lambda \in \overline{F(z)}$ with ord $\lambda \neq 0$. Then the element $x-\lambda$ of $\Omega^{\prime}$ is invertible, and for all $k \geq 1,(x-\lambda)^{-k}=\sum_{-\infty}^{\infty} a_{i} x^{i}$ in $\Omega^{\prime}$ with the $a_{i}$ in $\overline{F(z)}$. In particular, $l_{0}, l_{1}$ and $l_{-1}$ take each $(x-\lambda)^{-k}$ to an element of $\overline{F(z)}$.

Proof. If ord $\lambda>0, x-\lambda=x\left(1-\lambda x^{-1}\right)$ has inverse $x^{-1}\left(1+\lambda x^{-1}+\lambda^{2} x^{-2}+\cdots\right)$, while if ord $\lambda<0, x-\lambda=-\lambda\left(1-\lambda^{-1} x\right)$ has inverse $-\lambda^{-1}\left(1+\lambda^{-1} x+\lambda^{-2} x^{2}+\cdots\right)$.

Lemma 3.7. Let $U_{1}$ and $U_{2}$ be elements of $F[z, x]$. Suppose that $U_{2} \equiv x^{s} \bmod z$ for some s. Then $U_{2}$ has an inverse in $F\left[x, x^{-1}\right] \underline{[[z]]}$ and the coefficients of $x^{0}, x^{1}$ and $x^{-1}$ in the element $U_{1} U_{2}^{-1}$ of $F\left[x, x^{-1}\right][[z]]$ all lie in $\overline{F(z)}$.

Proof. Write $U_{2}$ as $x^{s}(1-z p)$ with $p$ in $F\left[x, x^{-1}, z\right]$. Then $x^{-s}\left(1+z p+z^{2} p^{2}+\cdots\right)$ is the desired inverse of $U_{2}$. If $\lambda$ in $\Omega$ has ord 0 then $1-z p\left(\lambda, \lambda^{-1}, z\right)$ has ord 0 and cannot be 0 . So when we factor $U_{2}$ in $\overline{F(z)}[x]$ as $q \cdot \Pi\left(x-\lambda_{i}\right)^{c_{i}}$ with $q$ in $F[z]$ and $\lambda_{i}$ in $\overline{F(z)}$, no ord $\left(\lambda_{i}\right)$ can be 0 . View $U_{1} U_{2}^{-1}$ as an element of $\overline{F(z)}(x)$. As such it is an $\overline{F(z)}$ linear combination of powers of $x$ and powers of the $\left(x-\lambda_{i}\right)^{-1}$. Since $l_{0}, l_{1}$ and $l_{-1}$ are $\Omega$-linear they are $\overline{F(z)}$-linear. Lemma 3.6 then tells us that $U_{1} U_{2}^{-1}$, viewed as an element of $\Omega^{\prime}$, is mapped by each of $l_{0}, l_{1}$ and $l_{-1}$ to an element of $\overline{F(z)}$. This completes the proof.

Lemma 3.8. Let $A, B$ and $C$ be in $M_{s}(F)$ and $u \in F\left[x, x^{-1}\right][[z]]$ be an entry in the matrix $\left(I_{s}-z\left(A x+B+C x^{-1}\right)\right)^{-1}$. Then the coefficients of $x^{0}, x^{1}$ and $x^{-1}$ in $u$ all lie in $\overline{F(z)}$. 
Proof. $u$ may be written as $U_{1} / U_{2}$ where $U_{1}$ and $U_{2}$ are in $F[z, x]$ and $U_{2}=$ $\operatorname{det}\left(x I_{s}-z\left(A x^{2}+B x+C\right)\right)$. Then $U_{2} \equiv x^{s} \bmod z$, and we apply Lemma 3.7.

Corollary 3.9. If $\Lambda=M_{s}(F), F$ a field, then the matrix entries of $M_{0}, M_{1}$ and $M_{-1}$ are algebraic over $F(z)$. (So by Theorem 3.5 the same is true of the matrix entries of $G(w)$.)

Proof. $\left(I_{s}-z\left(A x+B+C x^{-1}\right)\right)^{-1}=\sum_{0}^{\infty}\left(A x+B+C x^{-1}\right)^{n} z^{n}$, and we combine Lemma 3.8 with Corollary 3.3.

\section{Examples}

Example 4.1. For $i, j$ positive integers define $v_{i, j}$ by:

(1) $v_{i, j}=1$ if $i-j \in\{-1,0,1\}$.

(2) $v_{i, j}=1$ if $j=i+3$ and $i$ is odd.

(3) All other $v_{i, j}$ are 0 .

We calculate $G(V)$ where $V=\left|v_{i, j}\right|$. If we take $s=2,(1)$ and (2) in the corollary to Theorem I are satisfied, and $D=B=\left(\begin{array}{ll}1 & 1 \\ 1 & 1\end{array}\right), A=\left(\begin{array}{ll}0 & 1 \\ 0 & 0\end{array}\right), C=\left(\begin{array}{ll}0 & 1 \\ 1 & 0\end{array}\right)$. Let $G=G(w)=$ $G\left(w^{*}\right)$. $G$ is a 2 by 2 matrix $\left(\begin{array}{c}g_{1} \\ g_{3} \\ g_{4}\end{array}\right)$ with entries in $F[[z]]$, and $g_{1}=G(V)$. By Corollary 2.8, $C G A G z^{2}+B G z-G+I_{2}=0$. Two of the four equations this gives are:

$$
\begin{array}{r}
z^{2} g_{1} g_{3}+z\left(g_{1}+g_{3}\right)-g_{3}=0 \\
z^{2} g_{3}^{2}+z\left(g_{1}+g_{3}\right)-g_{1}+1=0
\end{array}
$$

Solving the first equation for $g_{3}$ and substituting in the second we find that $G(V)=g_{1}$ is a root of:

$$
\left(z^{5}-z^{4}\right) x^{3}+\left(3 z^{4}-4 z^{3}+2 z^{2}\right) x^{2}+\left(2 z^{3}-4 z^{2}+3 z-1\right) x+\left(z^{2}-2 z+1\right)=0 .
$$

Example 4.2. For $i, j$ positive integers define $v_{i, j}$ by:

(1) $v_{i, j}=1$ if $i-j \in\{-1,0,1\}$.

(2) $v_{i, j}=1$ if $j=i+3$ and $i$ is even.

(3) All other $v_{i, j}$ are 0.

We calculate $G(V)$ where $V=\left|v_{i, j}\right|$. Since $v_{2,5}=1$, condition (1) of the corollary to Theorem $I$ is not met when $s=2$, and we instead take $s=4$.

Now

$$
D=B=\left(\begin{array}{llll}
1 & 1 & 0 & 0 \\
1 & 1 & 1 & 0 \\
0 & 1 & 1 \\
0 & 0 & 1 & 1
\end{array}\right) \quad A=\left(\begin{array}{llll}
0 & 0 & 0 & 1 \\
0 & 0 & 0 & 0 \\
0 & 0 & 0 & 0 \\
0 & 0 & 0 & 0
\end{array}\right) \quad \text { and } \quad C=\left(\begin{array}{llll}
0 & 0 & 0 & 0 \\
1 & 0 & 0 & 0 \\
0 & 0 & 0 & 0 \\
1 & 0 & 1 & 0
\end{array}\right)
$$


Let the entries in the first column of the 4 by 4 matrix $G=G(w)$ be $a, b, c$ and $d$. Examining the entries in the first column of the matrix equation $G=B G z+C G A G z^{2}+I_{4}$ we see:

$$
\begin{aligned}
a & =(a+b) z+1 \\
b & =(a+b+c) z+b d z^{2} \\
c & =(b+c+d) z \\
d & =(c+d) z+d(a+c) z^{2}
\end{aligned}
$$

Using Maple to eliminate $b, c$, and $d$ from this system we find that $a=G\left(V^{*}\right)$ is a root of:

$$
\begin{aligned}
&\left(z^{2}\right) \cdot(z-1)^{3} \cdot\left(3 z^{2}+3 z-2\right) \cdot x^{3} \\
&+(z-1)^{2} \cdot\left(9 z^{4}+6 z^{3}-11 z^{2}+5 z-1\right) \cdot x^{2} \\
&+(2 z-1) \cdot\left(5 z^{4}-13 z^{2}+9 z-2\right) \cdot x \\
&+(2 z-1)^{2} \cdot\left(z^{2}+2 z-1\right)=0 .
\end{aligned}
$$

Example 4.3. For $i, j$ positive integers define $v_{i, j}$ by:

(1) $v_{i, j}=1$ if $i-j \in\{-1,1\}$.

(2) $v_{i, j}=1$ if $i-j \in\{-3,3\}$ and $i \equiv 2(\bmod 3)$.

(3) All other $v_{i, j}$ are 0 .

We calculate $G(V)$ where $V=\left|v_{i, j}\right|$. Take $s=3$. Then:

$$
A=\left(\begin{array}{lll}
0 & 0 & 1 \\
0 & 1 & 0 \\
0 & 0 & 0
\end{array}\right) \quad B=D=\left(\begin{array}{lll}
0 & 1 & 0 \\
1 & 0 & 1 \\
0 & 1 & 0
\end{array}\right) \quad C=\left(\begin{array}{lll}
0 & 0 & 0 \\
0 & 1 & 0 \\
1 & 0 & 0
\end{array}\right)
$$

The determinant of the matrix $x I_{3}-z\left(A x^{2}+B x+C\right)$ is $-x^{2}\left(z x^{2}+\left(3 z^{2}-1\right) x+z\right)$. The splitting field of this polynomial over $F(z)$ is the extension of $F(z)$ generated by $\sqrt{1-10 z^{2}+9 z^{4}}$. The arguments of section 3 show that $M_{0}, M_{1}$ and $M_{-1}$ have entries in this extension field. It's not hard to write down these matrices explicitly using the partialfraction decomposition argument. Theorem 3.5 and a Maple calculation then show that the $(1,1)$ entry in $G(w)$ is $4 /\left(3+z^{2}+\sqrt{1-10 z^{2}+9 z^{4}}\right)$. Since $D=B, G\left(w^{*}\right)=G(w)$, and this $(1,1)$ entry is the desired $G(V)$.

\section{$5 \quad$ More algebraic generating functions}

Definition 5.1. Suppose that $\Lambda=M_{s}(F), F$ a field, and that $A, B, C, D$ are in $\Lambda$. Then $\mathcal{L} \subset$ the field of fractions of $F[[z]]$ is the extension field of $F(z)$ generated by the matrix entries of the $M_{0}, M_{1}$ and $M_{-1}$ of Definition 3.2. 
Remark 5.2. As we've seen $\mathcal{L}$ contains the matrix entries of $G(w)$ and $G\left(w^{*}\right)$ and is finite over $F(z)$. Indeed the proofs of Lemmas 3.7, 3.8 and Corollary 3.9 show that $\mathcal{L} \subset$ a splitting field over $F(z)$ of the polynomial det $\left|x I_{s}-z\left(A x^{2}+B x+C\right)\right|$. One can say a bit more. The above polynomial splits into linear factors in $\Omega[x]$, and one may view its splitting field as a subfield of the valued field $\Omega$. By examining the partial-fraction decomposition one finds that $\mathcal{L}$ is fixed elementwise by each automorphism of the splitting field that is the identity on $F(z)$ and permutes the roots that have positive ord among themselves.

The goal of this section is to show that some generating functions related to $G(w)$ also have their matrix entries in $\mathcal{L}$. These results are used in [3] to show the algebraicity (under a conjecture) of certain Hilbert-Kunz series and Hilbert-Kunz multiplicities; see Theorems 3.1 and 3.4 of that note.

Now let $u_{k}^{(n)}$ be as in Lemma 2.12 where $k$ is a positive integer. By definition, $G^{*}(w)=$ $\sum u_{1}^{(n)} z^{n}$.

Lemma 5.3. $\sum_{n} u_{k+1}^{(n)} z^{n}=G(w)(A z) \sum_{n} u_{k}^{(n)} z^{n}$.

Proof. A standard walk from $k$ to 0 can be written in just one way as the concatenation of a standard walk from $k$ to $k$, the walk $(k, k-1)$ and a standard walk from $k-1$ to 0 .

Corollary 5.4. Fix $k \geq 1$. The generating function arising from the $(k, 1)$ entries of the matrices $W^{n}$ has its matrix entries in $\mathcal{L}$.

Proof. Corollary 2.13 shows that this generating function is $\sum_{n} u_{k}^{(n)} z^{n}$, and we use Lemma 5.3 and induction.

Definition 5.5. $G_{r}^{*}=\sum\left(\begin{array}{c}\alpha_{0} \\ r\end{array}\right) w^{*}(\alpha) z^{l(\alpha)}$, the sum extending over all standard walks finishing at 0 .

Evidently $G_{0}^{*}=\sum_{k=0}^{\infty} \sum_{n=0}^{\infty} u_{k+1}^{(n)} z^{n}$. By Lemma 5.3, this is

$$
\left(1+G(w) A z+(G(w) A z)^{2}+\cdots\right) G\left(w^{*}\right) .
$$

So:

Lemma 5.6. $(1-G(w) A z) G_{0}^{*}=G\left(w^{*}\right)$.

A variant of this is:

Lemma 5.7. $(1-G(w) A z) G_{r+1}^{*}=G(w)(A z) G_{r}^{*}$.

Proof. We introduce new weight functions $w \mid t$ and $w^{*} \mid t$ as follows. Replace $\Lambda, A$ and $C$ by $\Lambda[[t]], A(1+t)$ and $C(1+t)^{-1}$, and let $w \mid t$ and $w^{*} \mid t$ be the new $w$ and $w^{*}$ that arise. If $\alpha=\left(\alpha_{0}, \ldots, \alpha_{l}\right)$ is a walk from $k$ to 0 then there are $k=\alpha_{0}$ more steps of size -1 in the walk than there are steps of size 1 . It follows that $w \mid t(\alpha)$ and $w^{*} \mid t(\alpha)$ are $(1+t)^{\alpha_{0}} w(\alpha)$ and 
$(1+t)^{\alpha_{0}} w^{*}(\alpha)$. In particular, $G(w \mid t)=G(w)$ and $G\left(w^{*} \mid t\right)=G\left(w^{*}\right)$. Applying Lemma 5.6 in this new situation we find:

$$
((1-G(w) A z)-G(w) A z t)\left(\sum_{k=0}^{\infty} \sum_{n=0}^{\infty}(1+t)^{k} u_{k+1}^{(n)} z^{n}\right)=G\left(w^{*}\right) .
$$

In particular, the coefficient of $t^{r+1}$ in the left-hand side of the above equation is 0 . Evaluating this coefficient we get the lemma.

Theorem 5.8. Let $a_{1}, a_{2}, \ldots$ be elements of $F$. Suppose there is a polynomial function whose value at $j$ is $a_{j}$ for sufficiently large $j$. Let $R_{n}=\sum_{1}^{\infty} a_{k} u_{k}^{(n)}$. Then all the matrix entries of $\sum R_{n} z^{n}$ lie in $\mathcal{L}$.

Proof. Corollary 5.4 shows that the generating function arising from any single $(j, 1)$ entry has matrix entries in $\mathcal{L}$. So we may assume that $j \rightarrow a_{j}$ is a polynomial function. Since any polynomial function is an $F$-linear combination of the functions $j \rightarrow\left(\begin{array}{c}j-1 \\ r\end{array}\right)$, $r=0,1,2, \ldots$ we may assume $a_{j}=\left(\begin{array}{c}j-1 \\ r\end{array}\right)$. But then $\sum R_{n} z^{n}$ is $G_{r}^{*}$, and we use Lemmas 5.6, 5.7 and induction.

Corollary 5.9. Suppose $V=\left|v_{i, j}\right|, i, j \geq 1$ is a matrix with entries in $F$ satisfying:

(1) $v_{i, j}=0$ whenever $i \leq s$ and $j>2 s$ or $j \leq s$ and $i>2 s$.

(2) $v_{i+s, j+s}=v_{i, j}$ whenever $i+j \geq s+2$.

(3) The initial $2 s$ by $2 s$ block in $V$ is $\left(\begin{array}{cc}D & C \\ A & B\end{array}\right)$.

Suppose further that $a_{1}, a_{2}, \ldots$ are in $F$ and that for each $i, 1 \leq i \leq s$, there is a polynomial function agreeing with $k \rightarrow a_{i+s k}$ for large $k$. Let $v_{i}^{(n)}$ be the $(i, \overline{1})$ entry in $V^{n}$. Then $\sum_{i, n} v_{i}^{(n)} a_{i} z^{n}$ is in $\mathcal{L}$.

Proof. Construct $W$ as in the proof of the corollary to Theorem I. As the first column of $W^{n}$ is $u_{1}^{(n)}, u_{2}^{(n)}, \ldots$ it follows that $v_{i+s k}^{(n)}$ is just the $(i, 1)$ entry in the $s$ by $s$ matrix $u_{k+1}^{(n)}$. Theorem 5.8 shows that for each $i$ with $1 \leq i \leq s, \sum_{k, n} v_{i+s k}^{(n)} a_{i+s k} z^{n}$ is in $\mathcal{L}$. Summing over $i$ we get the result.

The following results may seem artificial but they're what we need for the applications to Hilbert-Kunz theory in [3].

Lemma 5.10. Let $Y$ be a finite dimensional vector space over $F, T: Y \rightarrow Y$ and $l: Y \rightarrow F$ linear maps and $y_{1}, y_{2}, \ldots$ a sequence in $Y$. Let $V$ and $s$ be as in Corollary 5.9. Suppose that for each $i, 1 \leq i \leq s$, each co-ordinate of $y_{i+s k}$ with respect to a fixed basis of $Y$ is an eventually polynomial function of $k$. Define $y^{(n)}$ inductively by $y^{(0)}=0$, $y^{(n+1)}=T y^{(n)}+\sum v_{i}^{(n)} y_{i}$ - see Corollary 5.9 for the definition of $v_{i}^{(n)}$. Then $\sum l\left(y^{(n)}\right) z^{n}$ is in $\mathcal{L}$. 
Proof. $(I-z T) \sum y^{(n)} z^{n}=\sum_{i, n} v_{i}^{(n)} y_{i} z^{n+1}$. By Corollary 5.9, all the co-ordinates of $(I-z T) \sum y^{(n)} z^{n}$ with respect to a fixed basis of $Y$ lie in $\mathcal{L}$. Since $\operatorname{det}|I-z T|$ is a non-zero element of $F(z) \subset \mathcal{L}$, the same is true of the co-ordinates of $\sum y^{(n)} z^{n}$, giving the lemma.

Theorem 5.11. Suppose $X$ is a vector space over $F, Y$ is a finite dimensional subspace, $T: X \rightarrow X$ is linear with $T(Y) \subset Y$, and $E_{1}, E_{2}, \ldots$ lie in $X$. Suppose further that $T\left(E_{j}\right)=\sum v_{i, j} E_{i}+y_{j}$, where $V=\left|v_{i, j}\right|$ is as in Lemma 5.10 and $y_{1}, y_{2}, \ldots$ is a sequence in $Y$ satisfying the condition of Lemma 5.10. Then if $l: X \rightarrow F$ is linear with each $l\left(E_{i}\right)=0$, the power series $\sum_{0}^{\infty} l\left(T^{n}\left(E_{1}\right)\right) z^{n}$ is in $\mathcal{L}$.

Proof. Define $y^{(n)}$ as in Lemma 5.10. Using the identity $\sum_{j} v_{i, j} v_{j}^{(n)}=v_{i}^{(n+1)}$ and induction we find that $T^{n}\left(E_{1}\right)=\sum v_{i}^{(n)} E_{i}+y^{(n)}$. So $l\left(T^{n}\left(E_{1}\right)\right)=l\left(y^{(n)}\right)$ and we apply Lemma 5.10 .

The following example is closely related to our calculations in [2]. We explain how this and similar examples relate to Hilbert-Kunz theory in [3].

Example 5.12. Suppose $\delta_{1}$ and $\delta_{2}$ are a basis of $Y$, that $y_{1}=6 \delta_{1}$ and that $y_{k}=(8 k-$ 2) $\delta_{1}+\delta_{2}, k>1$. Suppose further that $T\left(\delta_{1}\right)=16 \delta_{1}, T\left(\delta_{2}\right)=4 \delta_{1}+4 \delta_{2}, T\left(E_{1}\right)=$ $E_{1}+E_{2}+y_{1}$, and that $T\left(E_{k}\right)=E_{k-1}+E_{k+1}+y_{k}$ for $k>1$. Suppose $l: X \rightarrow F$ takes $\delta_{1}$ to 1 , and $\delta_{2}$ and each $E_{k}$ to 0 . We shall calculate the power series $S=\sum l\left(T^{n}\left(E_{1}\right)\right) z^{n}$ explicitly. (Theorem 2.4 of [3] and the observation following it arise from our formula for S.)

In the above situation, $v_{1,1}=v_{i, i+1}=v_{i+1, i}=1$ and all other $v_{i, j}$ are 0 . So we can take $s=1, A=C=D=1$ and $B=0$. Since $s=1, v_{k}^{(n)}=u_{k}^{(n)}$. It follows from this and the definition of the $y_{k}$ that $\sum_{k, n} v_{k}^{(n)} y_{k} z^{n+1}=z\left(8 G_{1}^{*}+6 G_{0}^{*}\right) \delta_{1}+z\left(G_{0}^{*}-G\left(w^{*}\right)\right) \delta_{2}$.

Now the matrix of $T: Y \rightarrow Y$ on the basis $\left(\delta_{1}, \delta_{2}\right)$ is $\left(\begin{array}{c}16 \\ 0\end{array} 4\right)$. It follows that the matrix of $I-z T$ is $\left(\begin{array}{ccc}1-16 z & -4 z \\ 0 & 1-4 z\end{array}\right)$ with inverse $\frac{1}{(1-16 z)(1-4 z)}\left(\begin{array}{cc}1-4 z & 4 z \\ 0 & 1-16 z\end{array}\right)$. Since $S$ is the coefficient of $\delta_{1}$ in $\sum l\left(y^{(n)}\right) z^{n}=(I-z T)^{-1} \cdot \sum_{k, n} v_{k}^{(n)} y_{k} z^{n+1}$, the last paragraph shows that $(1-16 z)(1-4 z) S=\left(z-4 z^{2}\right)\left(8 G_{1}^{*}+6 G_{0}^{*}\right)+4 z^{2}\left(G_{0}^{*}-G\left(w^{*}\right)\right)$. It only remains to calculate $G\left(w^{*}\right), G_{0}^{*}$ and $G_{1}^{*}$.

Lemma 2.7 and Corollary 2.8 show that $H(w)=z^{2} G(w)$, and $z^{2} G(w)^{2}-G(w)+1=$ 0 . So $G(w)$ and $H(w)$ are $\frac{1-\sqrt{1-4 z^{2}}}{2 z^{2}}$ and $\frac{1-\sqrt{1-4 z^{2}}}{2}$. Lemma 2.10 then shows $G\left(w^{*}\right)=$ $\frac{1}{2 z(1-2 z)}\left(-1+2 z+\sqrt{1-4 z^{2}}\right)$. Making use of Lemmas 5.6 and 5.7 we find that $G_{0}^{*}$ and $G_{1}^{*}$ are $\frac{1}{1-2 z}$ and $\frac{1}{2(1-2 z)^{2}}\left(-1+2 z+\sqrt{1-4 z^{2}}\right)$. A brief calculation then gives the explicit formula:

$$
(1-16 z)(1-4 z)(1-2 z)^{2} S=4 z(1-2 z)^{2}+\left(2 z-12 z^{2}\right) \sqrt{1-4 z^{2}} .
$$




\section{References}

[1] I. Gessel, A factorization for formal Laurent series and lattice path enumeration, J. Combinatorial Theory Ser A 28 (1980), 321-337.

[2] P. Monsky, Rationality of Hilbert-Kunz multiplicities: a likely counterexample, Michigan Math. J. 57 (2008), 605-613.

[3] P. Monsky, Algebraicity of some Hilbert-Kunz multiplicities (modulo a conjecture), Preprint 2009, arXiv: math. AC/0907.2470 